Política y Sociedad

ISSN: $\quad 1130-8001$

ISSN-e: $1988-3129$

\title{
La invención de los objetos: deporte, estandarización y subjetividad moderna
}

\author{
Olatz González Abrisketa ${ }^{1}$
}

Recibido: 14-11-2019 / Aceptado: 31-07-2020

Resumen. Este artículo aborda la naturaleza apática que la modernidad ha atribuido a los objetos. Se centra en la estandarización de los mismos en el deporte y ofrece un caso anómalo dentro del mismo: el de la pelota vasca. A pesar de la regulación de sus límites, en dimensiones y peso, las pelotas en el juego vasco son singulares, es decir, cada una de ellas posee una personalidad propia que debe ser reconocida. Tres días antes del partido, los pelotaris se reúnen para elegir las pelotas y mostrarlas al público, un acontecimiento que abre un juego de perspectivas que tiene efectos en la conceptualización de pelotas y pelotaris y que dificulta considerarlos objetos y sujetos respectivamente. Haciendo uso de conceptos como cuasi-objeto (Michel Serres) o reciprocidad de perspectivas (Roy Wagner), el artículo defiende que la "invención de los objetos" producida por la modernidad niega procesos de reconocimiento con las cosas que son sustanciales para la comprensión del sí-mismo y de los otros. Los "sujetos" sostienen la ilusión de que son ellos, y nada más ellos, los que están dirimiendo sus propias diferencias y obvian el hecho de que son a la vez objeto y sujeto para el otro, sea este persona, animal o cosa.

Palabras clave: antropología; deporte; pelota vasca; pelotas; cuasi-objetos; estandarización; subjetividad; enactuación; perspectivismo; reciprocidad de perspectivas.

\section{[en] Invention of Objects: Sports, Standardization and Modern Subjectivity}

Abstract. This article addresses the apathetic nature modernity attributes to objects. It focuses on their standardization in sport and offers an anomalous case within it: that of the Basque pelota. Despite the regulation of their limits, in size and weight, the balls in the Basque game are unique, that is, each one of them has its own personality and this must be recognized. Three days before the game, the pelotaris meet to choose the balls and show them to the public, an event that opens up a game of perspectives that has an effect on the conceptualization of balls and pelotaris and makes it difficult to consider them objects and subjects respectively. Making use of concepts such as quasi-object (Michel Serres) or reciprocity of perspectives (Roy Wagner), the article argues that the "invention of objects" produced by modernity denies processes of recognition with things that are substantial for the understanding of the self and others. The "subjects" hold the illusion that they, and only they, settle their own differences and ignore the fact that are both object and subject for the other, be it person, animal or thing.

Keywords: anthropology; sports; basque pelota; balls; quasi-objects; standarization; subjectivity; enaction; perspectivism; reciprocity of perspectives.

Universidad del País Vasco (UPV/EHU) (España).

E-mail: olatz.gonzalez@ehu.eus 
Cómo citar: González Abrisketa, O. (2020): "La invención de los objetos: deporte, estandarización y subjetividad moderna", Politica y Sociedad, 57(2), pp. 395-414.

Sumario. 1. Introducción. 2. Las pelotas. 3. El pelotari. 4. El partido. 5. El mundo de la pelota. 6. Conclusión. 7. Bibliografía.

Agradecimientos. La investigación que fundamenta este artículo se ha realizado gracias al apoyo económico e intelectual del Grupo de Investigación consolidado Tipo A del Sistema Universitario Vasco. "Cambio social, formas emergentes de subjetividad e identidad en la sociedad contemporánea" [IT1199-19]. Quiero agradecer también a Iñaki Martínez de Albéniz su invitación a participar en este número y a los editores, Elena Urieta y Fernando García Selgas, por su buen hacer y constante atención. Este trabajo nunca hubiera sido posible sin aquellos que durante un tiempo me permitieron inmiscuirme en su trabajo y en sus vidas. Un agradecimiento especial para quienes me enseñaron a escuchar las pelotas: Fernando Tapia (Tapia I), Martín Alustiza, Juan Mari Juaristi (Atano XIII) y Roberto García Ariño (Ariño IV).

"Lo mismo que nuestra cosmología constructivista puede ser resumida en la fórmula saussureana: el punto de vista crea el objeto — siendo el sujeto la condición originaria fija de donde emana el punto de vista-, el perspectivismo amerindio procede según el principio de que el punto de vista crea el sujeto; será sujeto quien se encuentre activado o "accionado" por el punto de vista".

Eduardo Viveiros de Castro.

Perspectivismo y multinaturalismo en la América Indígena

\section{Introducción}

Una de las más agradables sorpresas de mi etnografía sobre la pelota a mano profesional en el País Vasco fue descubrir que las pelotas que se utilizan en el juego no están estandarizadas y que existe todo un proceso de selección y cuidado de las mismas. Desde su fabricación hasta que son jugadas en el frontón, cada vez que un partido profesional tiene lugar, decenas de personas - casi en su totalidad varones-- escudriñan y hablan de las pelotas durante horas y a lo largo de varios días. Las aprietan en sus manos para sentir su dureza y calibrar sus dimensiones, las golpean para identificar su exigencia y las botan para escuchar su sonoridad y predecir así su comportamiento. "La pelota es un ser vivo" es la sentencia que justifica todas las atenciones que reciben las pelotas. El carácter orgánico de sus materiales - goma, lana y cuero_- que se ven afectados por la humedad y la temperatura y cambian el comportamiento de la pelota, y la singularidad de cada una de ellas justifica el largo proceso de reconocimiento, selección y cuidado por el que pasan las pelotas hasta que llegan a protagonizar un partido.

Ciertamente las pelotas protagonizan el partido tanto como los pelotaris. Tres días antes del mismo, los pelotaris comparecen públicamente en el frontón para realizar "la selección del material", es decir, elegir las pelotas que pondrán en juego. Cada pelotari debe elegir sus dos (o tres pelotas si se trata de una pareja) de entre las diez previamente elegidas por el seleccionador del material. Este es una figura neutral que debe escoger diez de las veinte pelotas que los intendentes - contratados por la 
empresa de cada pelotari- le ofrecen y que han elegido después de haber probado más de cien esa misma mañana (Figura 1). Una vez elegidas las pelotas, el seleccionador custodia las cuatro (o seis) en una caja sellada hasta el día del partido. La prensa informará sobre las dimensiones y el peso de las pelotas elegidas y especulará sobre su carácter, sobre el que los pelotaris opinarán a su vez en rueda de prensa, dejando claro si están satisfechos o no con las opciones recibidas y explicando cómo afrontan el partido con ese cestaño o conjunto de pelotas. Esto influirá en las quinielas que preceden al partido y que marcarán la salida de la apuesta.

Figura 1. Pelotas que los intendentes llevan a probar al frontón el día de la selección de material

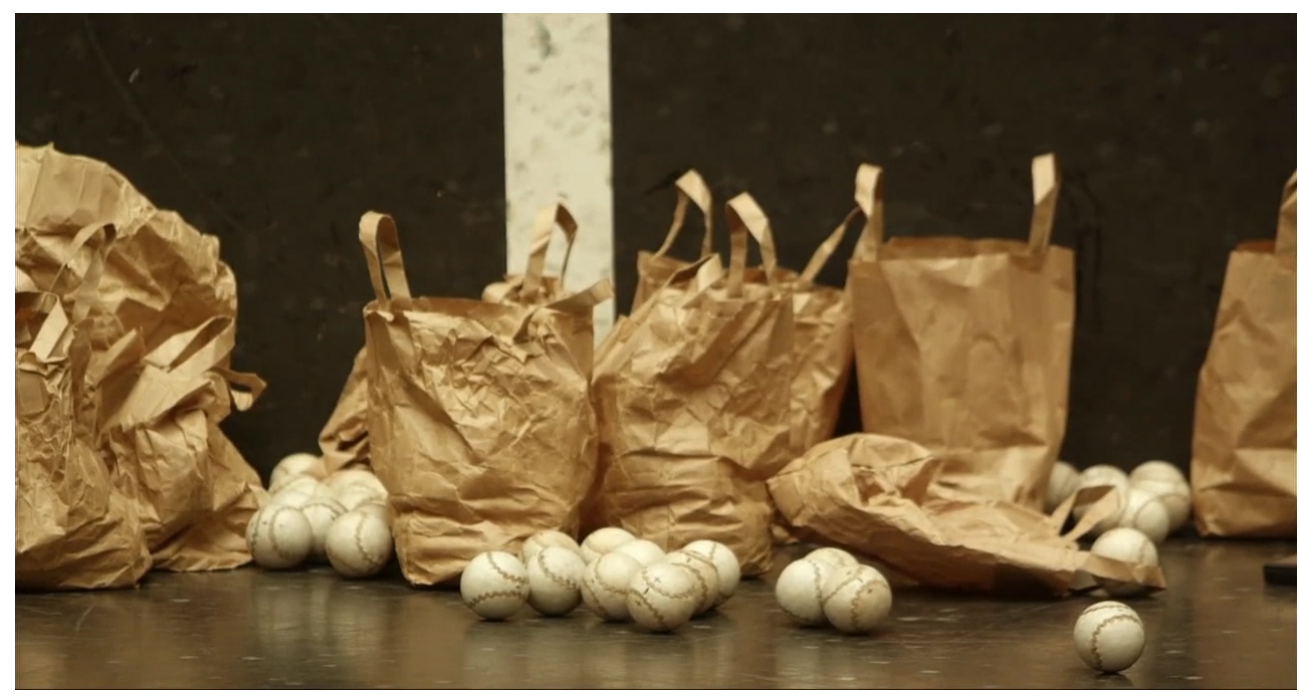

Fuente: fotograma de la película Pelota II (Jørgen Leth \& Olatz González Abrisketa, 2015).

Hace ahora 15 años interpretaba estos hechos apelando a la dimensión simbólica del objeto que da nombre al juego: la pelota. La incluía entre ese tipo de cosas que adquieren protagonismo para determinado grupo o comunidad por su capacidad para representarlo, por su capacidad para concretar aquello con lo que el grupo busca identificarse. Hablaba de las propiedades de la pelota "vasca", de su contundencia, de su dureza. También de lo que denominaba ética estética; la expresión de las virtudes referenciales encarnadas en el modo de golpear la pelota. La pelota en sí misma se convertía además en vehículo para la comprensión de la virtud "vasca" por excelencia: la nobleza. El bote recto, acorde al golpe del pelotari, es lo que define a una pelota como noble, ilustrando así ese valor altamente ensalzado. Pero, más allá de las capacidades representativas de la pelota, mis textos muestran una inquietud por dejar constancia de otra dimensión de las pelotas que no sabía cómo afrontar analíticamente. Ya en el primer párrafo del capítulo dedicado a las mismas hablaba de objetos que

"No se agotan en su conformación visible, en su fisicidad, sino que ofrecen un campo de posibilidad al sujeto que se topa con ellos. Son brotes de relación, de reversibilidad, de experiencia. Conforman el mundo y con él, (trans)forman al 
sujeto, puesto que le obligan a toparse con lo que está más allá de sí mismo. El encuentro que implican, la apertura que proponen al sujeto no es más que aquello que le hace humano: la relación, el intercambio". (Abrisketa, 2005: 311)

A pesar de esta clara apelación a la agencia de las pelotas, no tenía entonces las herramientas teóricas necesarias para abordar una revisión conceptual de la que había sido la tradición dominante durante mis estudios de licenciatura en antropología, liderada por Clifford Geertz, Victor Turner y Mary Douglas fundamentalmente. La pelea de gallos del primero me había animado a dedicar mi investigación doctoral a la pelota, y conceptos como ethos y cosmovisión, los símbolos rituales y las clasificaciones sociales inscritas en el cuerpo marcaban mi comprensión de lo que encontraba en el campo. Había sin embargo algo que se resistía. La atenta dedicación que se les procuraba a las pelotas me impedía reducirlas a simples metáforas, portadoras de algún significado independiente de propia materialidad, y por tanto mera ilustración de los intercambios simbólicos entre los sujetos. Decía entonces:

Durante el partido, la pelota es también indicador último del juego: establece el que un lance sea buena o falta, el que el tanto suba a un cartón u otro, el que sea un pelotari u otro el que saque, el que el momio se acerque o se distancie. Media entre los pelotaris define su ubicación en la cancha, motiva la actuación del juez, marca la apuesta y acompasa la emoción del público. La pelota es la batuta del partido. Dependiendo de su posición, los instrumentos se mantendrán expectantes o en pleno ejercicio. Todos los movimientos del frontón están determinados por el recorrido de aquella. "Al caer (terminaba, citando a Rilke), ordena como en una figura de danza a los jugadores" (Abrisketa, 2005: 312).

Esta centralidad de las pelotas en la coreografía que acontece en un frontón recuerda a la que traza Michel Serres en Le parasite para ejemplificar su teoría de los cuasiobjetos, híbridos que colapsan la distinción entre naturaleza y cultura, sujeto y objeto, individual y colectivo, material e ideal; más que ser una entidad, los cuasiobjetos fijan una posición, componen un mundo.

El balón no está ahí para el cuerpo; exactamente lo contrario es lo verdadero: el cuerpo es el objeto del balón; el sujeto se mueve alrededor de su sol. La habilidad con el balón se reconoce en el jugador que lo sigue y lo sirve en lugar de obligarlo a seguirlo y usarlo. Es el sujeto del cuerpo, sujeto de los cuerpos, y sujeto de los sujetos. Jugar es nada más que hacerse el atributo del balón como sustancia. Las leyes están escritas para él, definidas en relación con él, y nos doblegamos a estas leyes. La habilidad con el balón supone una revolución ptolemaica de la cual pocos teóricos son capaces, ya que están acostumbrados a ser sujetos en un mundo copernicano donde los objetos son esclavos (Serres, 1980: 303) ${ }^{2}$.

La emancipación — necesariamente parcial — de los objetos y las consecuencias que esta tiene en la formación y transformación de las subjetividades — modernas, y más que modernas - encuentra en el deporte un interesante campo de estudio

Todas las traducciones del original son propias. 
todavía escasamente explorado. Fascinados como estamos por la complejidad de los actantes e hibridaciones que nos presentan ciencia y tecnología, el deporte parece un ámbito demasiado literal, casi infantil, como para tomarlo en serio. El propio Bruno Latour, escapando de la senda de su maestro, afirma que "la pelota yendo de mano en mano es un pobre ejemplo de un cuasiobjeto, ya que, aunque traza el colectivo y el equipo no existiría sin ese testigo móvil, este no es modificado por los pases" (1996: 379). Latour da por hecho ese testigo móvil, pasando por alto las cuestiones de interés que lo han conformado ${ }^{3}$. No concibe que el deporte pueda ser otro de los sofisticados mecanismos a través de los cuales "progresivamente se oculta la relevancia que tienen los objetos en el interior del grupo o colectivo" (Tirado \& Maureira, 2016: 120). El mecanismo ha sido tan efectivo que hasta Latour lo ha pasado por alto; se denomina estandarización.

La estandarización de los materiales deportivos es un factor decisivo en el proceso de purificación y silenciamiento de los objetos que la modernidad llevó a cabo con el propósito de defender la excepcionalidad humana y, sobre todo, la de sus más dignos representantes, los varones blancos. Como sabemos, el deporte moderno ha sido una de las tecnologías más poderosas para salvaguardar la superioridad masculina ante la incorporación de las mujeres al trabajo remunerado (Messner, 1988). Los varones necesitaron constituir una esfera autónoma, que acreditara su preeminencia y justificara el mantenimiento de sus privilegios. La estandarización de los objetos deportivos es un componente más en la incubación de ese individuo autosuficiente (sic), emancipado de las constricciones materiales, que debe poder medirse en igualdad de condiciones con sus congéneres y hacerlo sin que nada se interponga en la valoración de su mérito.

La estandarización enmudece los objetos y los convierte en meros intermediarios, por continuar con la jerga latouriana. Frente a ello, la pelota vasca, que no ha vivido un proceso de estandarización análogo al del resto de juegos de pelota, se revela como un ejemplo significativo en el intento por restituir la agencia de los mediadores. Las pelotas en el juego vasco no solo no están calladas, sino que hablan. Aprender a escucharlas es el primer paso para reconocerlas. En la película Pelota II (Jørgen Leth \& Olatz González Abrisketa, 2015), una película que sigue la aventura de las pelotas desde que salen del taller del pelotero hasta que son jugadas en una final, hay una escena en la que el pelotari Retegi II, máximo campeón de la década de los 80 , compara el sonido de dos pelotas y afirma con resignación que una de ellas no vale para nada (Figura 2). El público ríe. No consigue percibir el matiz que permite al pelotari distinguirlas. Puedo asegurar, sin embargo, que las pelotas suenan diferente. Yo sentí que había dado un importante paso en la comprensión del juego de pelota cuando aprendí a oír las pelotas, a escucharlas. Puedo saber ahora si son pelotas nobles, buenas para jugar, si oigo un sonido que se propaga limpiamente y reverbera en todo el frontón, algo así como un "quissss". Y puedo saber, por el contrario, si son pelotas zakarrak, desperdicios, basura, si percibo un "clack", tal y como reproducía un intendente guiándome en la escucha. Estas parecen tener el núcleo roto cuando se botan. Puedo identificar las pelotas, sí, pero no puedo reconocerlas. Para conocer la personalidad de las pelotas, para saber cómo se van a comportar, es necesario jugarlas, actuarlas, y haber jugado muchas veces con ellas, haber aprendido cómo duelen, lo que cuesta moverlas. 
Figura 2. Retegi II y Retegi Bi comparan pelotas de 1983 y 2013

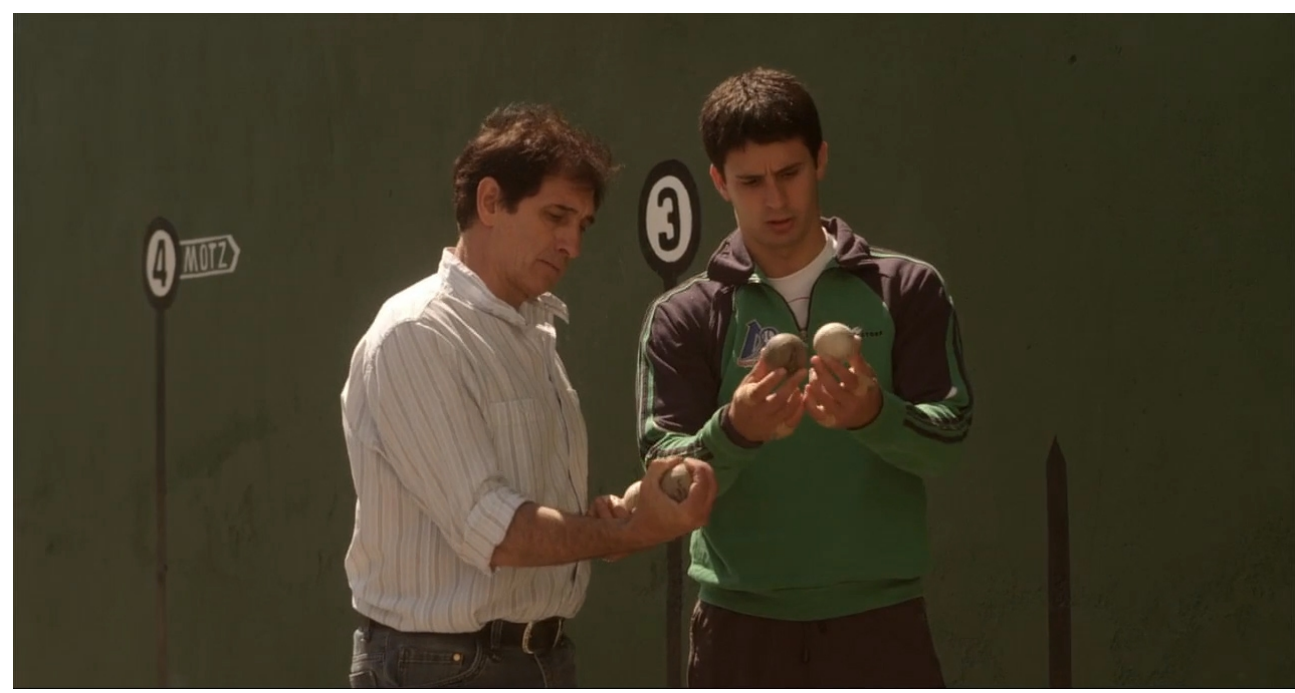

Fuente: fotograma de la película Pelota II (Jørgen Leth \& Olatz González Abrisketa, 2015).

La naturaleza apática que la modernidad ha atribuido a los objetos, y una de cuyas modulaciones ha sido la estandarización de los mismos, pretende ser aquí contrastada con el caso de las pelotas en el juego vasco, de quienes se dice que tienen personalidad propia. Mostrando lo que aporta al juego la singularidad de las pelotas, el artículo formula la pregunta ¿qué implicaciones tiene la estandarización de los objetos deportivos en la comprensión de uno mismo y lo que le rodea?, ¿qué formas de entender las relaciones sujeto-objeto se supeditan y cuáles se promocionan? El objetivo no es tanto considerar la agencia de las pelotas como entidades que otorgan existencia y durabilidad a lo social y que han sido omitidas del relato sociológico, ni siquiera desgranar la concepción emic que las reconoce como seres vivos, sino mostrar cómo pelotas y pelotaris mantienen una relación de mutua y dependiente cogeneración que revela una subjetividad ( $\mathrm{y}$ por tanto objetividad) que difiere de la promocionada por la modernidad.

Para defender lo primero, la mutua dependencia de pelotas y pelotaris, me voy a apoyar en el paradigma enactivo de las ciencias cognitivas, que en los últimos tiempos ha generado un renovado interés por la cognición dentro de la antropología (Ingold, 2000; Pedersen, 2007; Ishuu, 2012). Este paradigma, conocido también como el "4E paradigm", piensa la cognición como un proceso encorporado (embodied) ${ }^{4}$, encastrado (embedded), extendido (extended) y enactivo (enactive), en el que los objetos, así como otros seres, fuerzas y fenómenos, no ocupan una posición externa a la misma, sino que la generan en coacción con los cuerpos con los que entran en contacto. Para defender lo segundo, que la no estandarización esconde una concepción del vínculo sujeto-objeto distinta de la que ha

4 Como ya he justificado en otra parte (González-Abrisketa, 2013), considero que, siguiendo a Viveiros de Castro (2004), la traducción más apropiada para el verbo "to embody" al castellano es "encorporar". Desarrollamos los matices de esta diferenciación en Abrisketa \& Grados, 2018. 
promocionado la modernidad, haré uso de una tradición antropológica que podemos remontar a Claude Lévi-Strauss y su bricoleur, pero que ha enraizado a través de figuras como Roy Wagner, Eduardo Viveiros de Castro o Marilyn Strathern, referentes de lo que se ha denominado giro ontológico en antropología (Henare et al., 2007; Kohn, 2015; González-Abrisketa \& Carro-Ripalda, 2016). Los paralelismos que la relación entre pelotas y pelotaris encuentra en cosmovisiones otras y concepciones indígenas del sí mismo y del encuentro de este con otras cosas y seres nos ofrecerá una dimensión diferente de pensar los objetos, y que podría después de todo no ser tan extraña a contextos que consideramos modernizados.

\section{Las pelotas}

En la mayor parte de deportes que utilizan una pelota o balón - fútbol, baloncesto, tenis y demás-, las categorías de juego se miden por las competencias de las y los jugadores en relación a su fuerza, velocidad, visión de juego y técnica, entre otros. Desde los trece años en adelante, ambos sexos juegan con una pelota o balón estandarizados, los mismos con los que juegan las y los profesionales. En pelota no sucede así. El aumento de categoría implica un aumento en la dureza de la pelota, que se incrementa además con un aumento de peso, hasta llegar al máximo que puede alcanzar una pelota profesional para categoría masculina: 107 gramos.

La mayoría de personas que han jugado a pelota recuerdan su primer encuentro con ella como algo punzante, físicamente doloroso. A pesar de que las pelotas con las que juegan niñas y niños se denominan goxuak, "dulces", su dureza impacta con dolor en la mano de cualquier adulto que no esté acostumbrado a jugar. Las pelotas son, por su dureza, extremadamente exigentes, y obligan desde el principio a un entrenamiento continuado para poder dominarlas. No poder con la pelota es motivo frecuente para no aumentar la categoría. Sin embargo, esta impotencia no depende solo del peso de la pelota. El interior elástico de las mismas, de caucho, hace que posean más o menos bote y velocidad, y en definitiva mayor o menor violencia, de lo que depende el nivel de dificultad para dominarlas y moverlas con facilidad. Por ello, además de por las cualidades referidas arriba, el aumento de categoría va acompañado de una transformación del elemento de juego, que implica una constante adaptación por parte del o la pelotari. Hacerse "pelotari" implica un proceso de continua adaptación al progresivo aumento de la exigencia de la pelota.

A la variedad de pelotas para las distintas categorías y niveles, se suma el hecho de que cada pelota es única. Los materiales que componen la pelota, así como las condiciones de su fabricación, provocan que las pelotas difieran unas de otras, en ocasiones sustancialmente. Aprender a conocer y reconocer las pelotas es una de las principales competencias que deben adquirir los pelotaris, algo que distingue la pelota de aquellos deportes que han estandarizado sus elementos de juego y que podemos denominar - siguiendo a Allen Guttman- "modernos".

En el segundo capítulo de From Ritual to Record, Guttman establece los factores que caracterizan al deporte moderno: secularización, igualdad de oportunidades, especialización, racionalización, burocratización, cuantificación y búsqueda de 
récord (2004 [1978]). No ofrece Guttman una atención pormenorizada a la estandarización de los materiales deportivos, a la que se refiere someramente al explicar la racionalización; el establecimiento de reglas de juego que aseguren la igualdad de oportunidades y la universalidad del juego. La racionalización implica la estandarización, porque esta evita que las condiciones del juego cambien dependiendo del lugar donde se juegue, algo propio de los juegos premodernos, en los que las reglas se supeditaban a los espacios y costumbres locales. Permite así que el nivel de incertidumbre se reduzca y que las condiciones de juego se repliquen, aportando predecibilidad al deporte y permitiendo una gestión regulada del mismo.

Guttman reconoce que la evolución de los materiales deportivos ha transformado profundamente muchos deportes, pero no aborda las implicaciones de la estandarización de los objetos en el pensamiento moderno. Eliminar las diferencias entre los elementos en juego, además de reducir la incertidumbre, promueve una concepción individual de la valía personal. Los "sujetos" sostienen la ilusión de que son ellos, y nada más ellos, los que están dirimiendo sus propias diferencias. Esta uniformidad de los "mediadores", convertidos así en "intermediarios", encuentra un elocuente paralelismo en el análisis de Celia Amorós sobre la indiscernibilidad de las mujeres, lo que ella denomina "las idénticas" (1994; 2004). Frente a ellas, los varones se autoconstituyen como grupo juramentado de iguales, una igualdad que se sostiene sobre el reconocimiento y la distribución de las diferencias al interior del grupo, pero que solo es posible por exclusión y equiparación de aquellos a quienes se designa como "otros". Los integrantes del conjunto heterodesignado - las mujeres, los objetos - se sumergen así en el paisaje resignado de la indiferencia sobre el que los sujetos varones dirimen sus diferencias y pueden conceptualizarse como iguales.

La ficción humanista moderna de un sujeto autónomo que se reproduce gracias entre otros al ámbito deportivo se sostiene sobre dos clases de objetos: aquellos que acompañan la gesta deportiva del contendiente y aquellos que la dirimen. Denominaremos aliados a los primeros e imparciales a los segundos. Es sobre estos últimos sobre los que la estandarización ha sido más severa, a pesar de estar todos ellos regulados. Allen Guttman confunde ambos cuando da por hecho que incluso los "atletas griegos que competían directamente uno contra otro probablemente usaban el mismo disco o jabalina" (2004: 42-43). La mayoría de deportes mantienen una legión de técnicos que se encargan de mejorar las herramientas disponibles para proporcionar ventaja competitiva a sus jugadores, entre ellos el disco y la jabalina, precisamente. Las distintas calidades de los aliados — bicis, coches, pértigas o arcos- muestran que la igualdad de oportunidades no se sostiene sobre un cuerpo "desnudo", sino profusamente equipado. Estas herramientas deportivas, como máquinas, se convierten así en extensiones del cuerpo de las personas que las utilizan o para quienes han sido diseñadas, lo que sucede con las y los deportistas profesionales. Eso sí, más allá de estas prolongaciones, que no conculcarían la igualdad de oportunidades al entenderse que un sujeto se conforma junto a sus propiedades, lo que sostiene la ilusión de la ecuanimidad es la presencia de intermediarios, esos objetos imparciales que son para todos igual - en los casos mencionados arriba pistas, listones o dianas-. Balones y pelotas entrarían dentro de esta categoría de objetos que no pertenecen a nadie, que se dan por sentados $\mathrm{y}$, por tanto, permanecen en cierto sentido desactivados. 
En el caso del fútbol, por ejemplo, por intereses comerciales y publicitarios, cada competición genera su propio balón. La atención que recibe se limita a su estética, que está dirigida a lograr la aceptación popular y conseguir así su venta masiva. En ocasiones, alguna remesa provoca controversia por cuestiones técnicas, y eso da pie a que se analicen sus proporciones y cualidades materiales. Pero lo habitual es que el balón, una vez asegurada su funcionalidad, pase desapercibido. De ahí que sea posible sustituirlo por otro equivalente en el momento en que sale del campo. Solo en contacto con el sujeto, a través de firmas o adornos de diverso tipo, adquiere el balón cierta "personalidad". En pelota, por el contrario, la pelota es insustituible desde el momento en que ha sido seleccionada para el partido, y si este es trascendente, una final por ejemplo, la pelota será guardada y podrá acompañar a los trofeos (Figura 3).

Figura 3. Detalle de las pelotas de todas las finales que disputó Retegi II y que él guarda

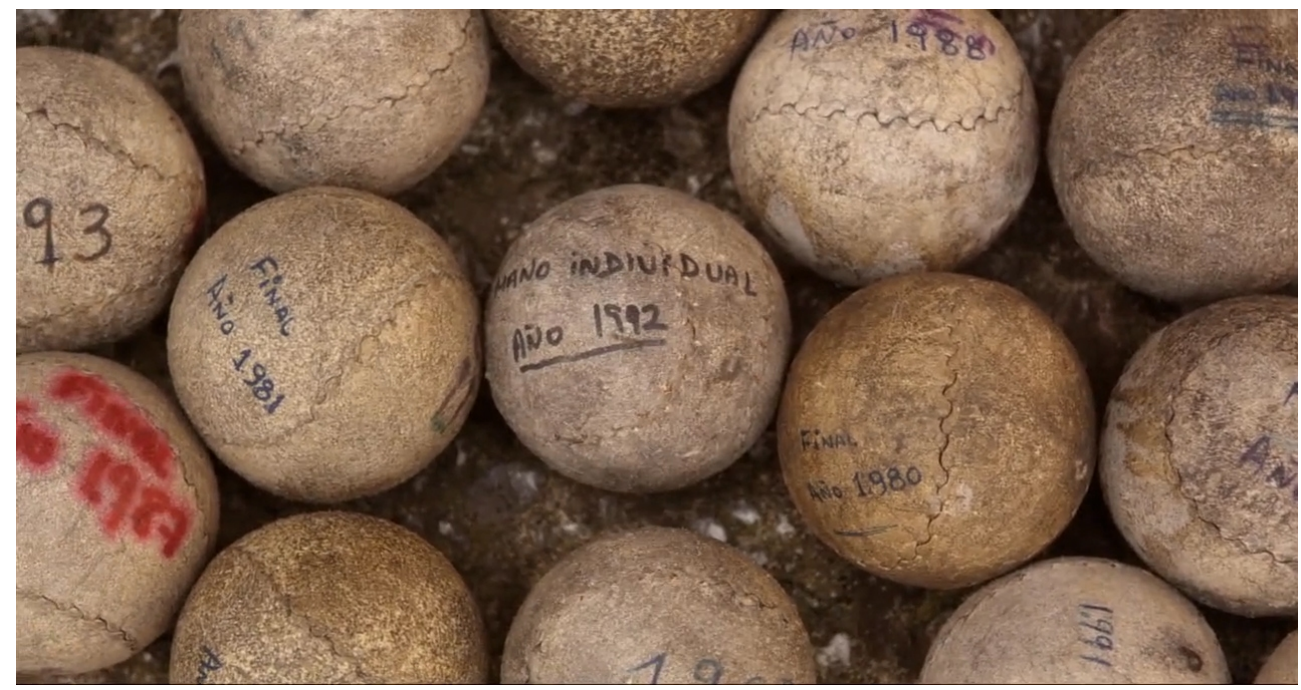

Fuente: fotograma de la película Pelota II (Jørgen Leth \& Olatz González Abrisketa, 2015).

Cada pelota posee una personalidad propia. Unas son más motelas (lentas), otras más ligeras, pueden ser botonas, secas, saltarinas, de más toque, más salida, o incluso ser más o menos pelota, es decir, más o menos exigente. Además, estas propiedades pueden intensificarse o cambiar si la pelota sufre algún tipo de tratamiento (aplicación de sebo, por ejemplo) o si es afectada por el calor o la humedad. La pelotas con las que juegan los profesionales varones deben ser de entre 61,50 y $63 \mathrm{~mm}$ de diámetro y 102 y 107 gramos de peso, pero dentro de esos parámetros, la variabilidad es infinita; tanta como pelotas hay.

Es una situación única en el mundo deportivo que los objetos imparciales puedan convertirse en aliados para alguna de las partes. La selección de material permite a los pelotaris elegir las pelotas que consideran más apropiadas para su juego. Esto tiene consecuencias en el modo en que se concibe el objeto y, por tanto, también en cómo se piensa el pelotari, algo que vamos a abordar en el siguiente apartado. 


\section{El pelotari}

Aprender a reconocer las pelotas es un proceso paralelo a hacerse pelotari, ya que no hay forma de que este proceso sea efectivo sin poner el cuerpo en ello, sin golpearlas, sin jugarlas. Al imaginar el encuentro con las pelotas, sin embargo, tendemos a pensar en un sujeto, el pelotari, con unas características específicas, que utiliza un instrumento, la pelota, por medio del cual puede exhibir sus habilidades. En el caso de la pelota vasca, además, las facultades del pelotari pueden ser favorecidas o perjudicadas dependiendo de la particularidad de la pelota elegida. Con esta imagen obviamos, sin embargo, el hecho inequívoco de que el pelotari no existe con anterioridad a la pelota. No hay posibilidad de ser ni reconocerse como pelotari sin el necesario contacto con la pelota, algo que podemos generalizar al resto de deportes y que podemos extender a todas las relaciones que establecemos con los objetos. Si, como afirma Fernando Domínguez Rubio, lo que diferencia las cosas de los objetos es que estos "están dotados de tipos particulares de valor, significado y poder" (2016: 82) que derivan de las prácticas y condiciones materiales y discursivas en las que se generan y reproducen, no es menos cierto que los sujetos dependen necesariamente de objetos para producir valor y significados y mantener determinadas relaciones de poder.

Esta necesaria interdependencia entre sujeto y objeto puede ser explicada a través de un concepto, la enactuación, que proviene de las ciencias cognitivas, pero que ha encontrado también su deriva en las ciencias sociales (Law \& Urry, 2004). Aunque utilizado ya en los años 60 por Jerome Bruner para describir el primero de los modos de representación del desarrollo cognitivo, aquel en el que el conocimiento se adquiere en la propia acción, el paradigma enactivo ha sido principalmente vinculado al neurobiólogo Francisco Varela. Muy cercana a la idea de mente en Gregory Bateson, en la que el sujeto no dirige, sino que participa de un continuo vital en el que está inmerso, Varela entiende la cognición como una acción encorporada (embodied action), una operación que se ejecuta con el cuerpo y en la que el que conoce y lo que es conocido mantienen una relación de mutua y dependiente cogeneración.

El paradigma enactivo de Varela pretende superar los acercamientos a la cognición en términos de representación, ya que toda representación implica un mundo preexistente, del que la mente (humana) descifra o interpreta sus características con el propósito de actuar a partir de las mismas. Varela rechaza este esquema del proceso cognitivo y defiende la cognición como acción encorporada en el contexto de la evolución como decurso natural: "La cognición ya no se encara como resolución de problemas a partir de representaciones; en cambio, la cognición en su sentido más abarcador consiste en la enactuación de un mundo - en hacer emerger un mundo- mediante una historia viable de acoplamiento estructural" (1992: 238).

Este paradigma permite a la antropología abordar la cognición humana en términos más acordes con las concepciones propias de las colectividades que estudia y conecta bien con las teorías que en los últimos años han provocado lo que se ha denominado el giro ontológico (Kohn, 2015; González-Abrisketa \& CarroRipalda, 2016). Un ejemplo de ello es el artículo de Morten Axel Pedersen sobre las ontologías chamanísticas del norte de Mongolia, que forma parte del volumen 
Thinking Through Things (Henare et al., 2007). Este libro propone una antropología dirigida a los objetos que asuma que las cosas dictan los términos de su propia significación, es decir, que son conceptos en sí y sin referencias fuera de sí, algo que colapsaría la distinción entre experiencia (material) y análisis (mental). Objetos y conceptos adquieren la misma categoría ontológica, lo que obliga a las y los investigadores a violentar las formas en que codifican y piensan sus materiales etnográficos. El artículo de Pedersen defiende que los artefactos de los chamanes del norte de Mongolia, lejos de ser meros complementos, constituyen andamios cognitivos con los que acceder a conocimientos religiosos de otro modo inaccesibles, algo que "permite explicar cómo un particular sentido Darhad del yo es enactuado a través de estas cosas chamánicas" (2007: 155).

En una línea semejante, aunque proponiendo pensar con las cosas, y no tanto a través de ellas, Miho Ishii confronta sus materiales etnográficos sobre espíritus y rituales de posesión en Ghana y el sur de la India con las revisiones que Hideo Kawamoto hace de la teoría de la autopoiesis de Maturana y Varela. Kawamoto, tal y como lo presenta Ishii, vincula la autopoiesis con los conceptos deleuzianos de intensidad y diferencia para defender que el sí mismo es aquello que se produce o emerge a través de la acción corporal: "Se relaciona con la noción de kinestesia, o del sentido práctico de 'ser capaz de hacer', más que con el sentido cognitivo de "conocer algo"' (2012: 374). En dicha emergencia, por tanto, las cosas ocupan un lugar central y necesario, ya que para que se produzca deben entran en relación de "coherencia" con las acciones que posibilitan y por tanto realizan. Es decir, en el proceso de realizar algo, de generar realidad a través de la acción, hay un movimiento conjunto entre las personas y los objetos en la que cada parte específica transforma y crea la acción de los otros y sus cualidades. Esta idea de coherencia deviene central en el trabajo de Ishuu, quien examina "la emergencia y formación de los mundos divinos en tanto que procesos autopoiéticos formados por las acciones coherentes de cuerpos y cosas" (2012: 373), y me sirve aquí para defender que el proceso de ajuste mutuo entre pelotaris y pelotas, la acción conjunta, no es un mero acoplamiento, sino que genera las partes, las hace emerger y en definitiva las realiza.

Esto no implica que el pelotari no puede proyectar antes del contacto y reconocimiento de la pelota su estrategia, algo que no entraría en confrontación con la consideración moderna del sujeto agentivo frente al objeto pasivo. Que el ser humano se provee de herramientas, y que estas son más o menos adecuadas para el propósito que se desea cumplir, es algo que forma parte del sentido común en el que nos hemos socializado. Aplicar la teoría enactiva al caso que nos ocupa, en cambio, obligaría a aceptar que el pelotari descubre su estrategia, mejor dicho, la compone, en el proceso de reconocer la pelota, momento de ajuste y búsqueda de coherencia entre ambos. Esta búsqueda no implica que no haya crisis, como apunta Ishii siguiendo a Kamawoto, más bien que esta es parte también del ajuste y emergencia de aquello que se es capaz de hacer o conocer.

Esta emergencia recuerda a la del bricoleur, quien actúa sin plan previo y que hace de los elementos a su alcance la fuente de su creatividad. Lévi-Strauss nos informa de que "el verbo bricoler se aplica al juego de pelota y de billar, a la caza y a la equitación, pero siempre para evocar un movimiento incidente: el de la pelota que rebota, el del perro que divaga, el del caballo que se aparta de la línea 
recta para evitar un obstáculo" (2002: 35). A diferencia del ingeniero o el científico, que actúan a partir de diseños o modelos preestablecidos, el bricoleur va generando su proyecto en conjunción con los elementos que encuentra, y cada nueva pieza le obligará a reorganizarlo. En el caso del pelotari, sin embargo, su proyecto no es algo que está más allá de sí mismo, sino que tiene que ver con su propia conformación como pelotari. Lo que proyectará no será una obra que, como la pelota, es de algún modo exterior a su cuerpo. Precisamente lo que le permite la pelota es descubrir sus propias habilidades, imaginar sus estrategias. Reconociendo las cualidades de esta, el pelotari se reorganiza a sí mismo, se reinventa.

La singularidad de la pelota permite por tanto al pelotari inventar posibilidades que no había contemplado antes, porque el conocimiento sobre lo que es capaz de hacer no se completa hasta que la pelota le otorga la oportunidad de que lo piense. El pelotari emerge a través del acto de mirarse desde las pelotas y ver con qué juego, quizás nunca imaginado antes, puede sacarles el mayor partido. Hacer suyas las pelotas no implica adaptarlas a las cualidades propias, sino descubrir las cualidades que aquellas potencian, que permiten actuar. Pensarse pelotari, literalmente quien juega con las pelotas, es asumir que las pelotas, por su singularidad, obligan a imaginarse una y otra vez de manera renovada.

Esta ecuación sin embargo no tiene sentido si en ella no incluimos a otro(s) pelotari(s). La pelota es un juego y como tal, siguiendo a Lévi-Strauss, es disyuntivo, hay dos partes que desde una supuesta simetría, o precisamente gracias a ella, como afirmaría René Girard, van a distinguirse, a dirimir quién gana y quién pierde, quién es ganador y quién víctima. En la relación pelota-pelotari siempre hay otro pelotari, esté o no presente. Esta triangulación es sobre la que versa el siguiente apartado.

\section{El partido}

Todo partido profesional de pelota se inicia, como ya hemos dicho, tres días antes de ser jugado, con la selección del material. En el mismo frontón en el que se va a disputar el partido, se convoca a los pelotaris para que elijan las pelotas con las que van a jugarlo. También está la prensa y público que se acerca para ver a los pelotaris y escuchar las pelotas. Ambos pelotaris están en el vestuario, colocándose los tacos: protecciones que evitan que la pelota impacte y dañe las zonas sensibles de sus manos. Están juntos, sentados uno al lado del otro, conversando amigablemente sobre cuestiones personales y/o deportivas (Figura 4). No hay tensión entre ellos, algo que cambiará una vez que hayan elegido las pelotas y la estrategia de cada uno haya quedado perfilada.

El seleccionador del material ya tiene las 10 pelotas que ofrecerá a los pelotaris y estos salen a la cancha para proceder a la elección. Primero lo hará el aspirante o, en el caso de que no haya un claro favorito, el que más tarde haya debutado. Tendrá 15 minutos para elegir sus dos pelotas. Con la ayuda de su botillero, la persona que le asesorará durante el partido, el pelotari prueba cada pelota, botándola repetidas veces y golpeándola contra el frontis, la pared frontal del frontón.

Los pelotaris siempre afrontan la selección del material con la esperanza de encontrar en el cestaño una pelota que "les entre bien en la mano". En mis trabajos 
anteriores sobre la relación entre pelota y pelotari comparaba este soñado acoplamiento perfecto con el del zapato de cenicienta o la espada de Arturo, objetos que extienden las capacidades de los cuerpos más allá de su piel. Esos objetos son de algún modo parte de sí, constituyen la particularidad del portador y exclusivo propietario. La pelota no es sin embargo un objeto aliado, ni tampoco uno completamente imparcial. Uno de los pelotaris la elige porque cree que le va a ser más favorable que el resto, pero eso no implica que la pelota le pertenezca. El oponente, una vez en el partido, podrá elegirla también para jugar e incluso ganarle con ella; una de las situaciones más desestabilizadoras que suceden con relativa frecuencia en pelota. Ganar con la pelota del contrario, o perder con la propia — su contraposición necesaria- es uno de los anhelos, y por lo tanto también temores, más extendidos entre los pelotaris.

Figura 4. Martínez de Irujo y Bengoetxea VI se colocan los tacos en el vestuario antes de la selección de material de la semifinal manomanista de 2013

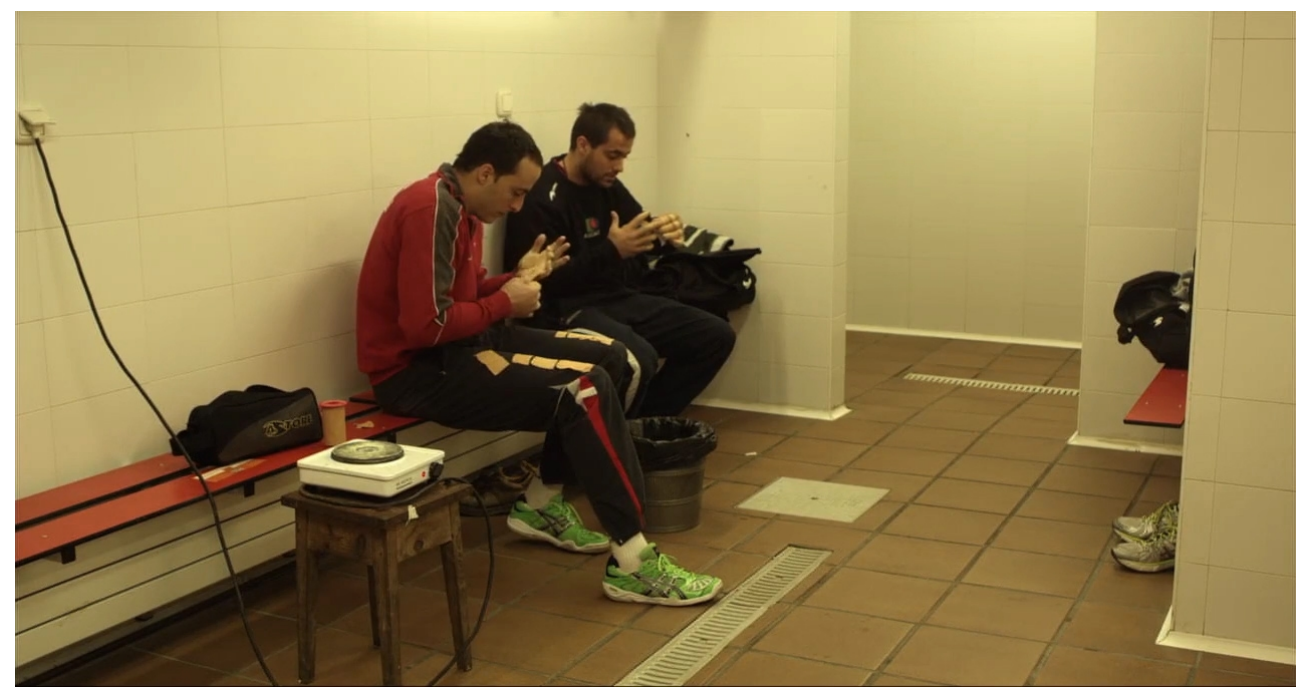

Fuente: fotograma de la película Pelota II (Jørgen Leth \& Olatz González Abrisketa, 2015).

Cuando el pelotari va ganando con facilidad se dice que "ha encontrado pelota". Se ha hecho con las posibilidades que ella ofrece y por eso es a través de ella que está dominando a su rival, haya sido él o no quien la ha elegido. Por eso, muchos pelotaris afrontan este momento con inquietud y preocupación, sobre todo los más inexpertos. Nunca parecen encontrar lo que están buscando, y más aún si se sienten inseguros ante el partido. La selección de material es el momento en que las sensaciones frente al partido se intensifican, por eso los veteranos aprovechan el momento para meter presión o jugar psicológicamente con el rival, presionándole para que se decida o haciendo ellos mismos una elección meteórica.

Una vez el primer pelotari ha elegido sus dos pelotas, y se las ha entregado al seleccionador, al favorito le quedarán ocho pelotas para hacer su selección. Dispondrá de otros 15 minutos y, como el anterior, se apoyará en su botillero para realizarla. Cuando el segundo pelotari tiene sus dos pelotas, el seleccionador 
entregará a cada uno las pelotas del oponente para que las prueben. Aquí empieza la escisión entre los pelotaris, el partido. Cada uno visualiza a través de las pelotas la estrategia del rival y empieza a conjeturar por qué ha elegido esas pelotas, cómo se ha imaginado y cómo le ha imaginado a él, cuáles son las cualidades propias que quiere potenciar y las ajenas que quiere neutralizar. Como los objetos heteróclitos que componen el tesoro del bricoleur, en la selección de pelotas, el pelotari interroga a las pelotas "para comprender lo que cada una de ellas podría 'significar"' (Lévi-Strauss, 2002: 38), es decir, las jugadas que cada una de ellas podría concretar.

El posado frente a los fotógrafos con la caja abierta, a pesar de las sonrisas, esconde ya cierta tensión. Cada uno se enfrenta a su imagen proyectada por el otro en esas pelotas. Su sí-mismo aparece cuestionado por la mirada del otro, que a su vez se interroga por la estrategia del rival. El lote de pelotas condensa y precede el encuentro "cara a cara". Al finalizar el posado, el seleccionador se lleva las pelotas para su pesado y el sellado de la caja. Las pelotas quedarán custodiadas por él hasta el partido, pero los pelotaris las tendrán muy presentes. Las armas de la contienda ya están elegidas y contienen todas las posibilidades. Alea jacta est. Los pelotaris vuelven al vestuario a quitarse los tacos y toda la conversación girará ahora en torno a las pelotas.

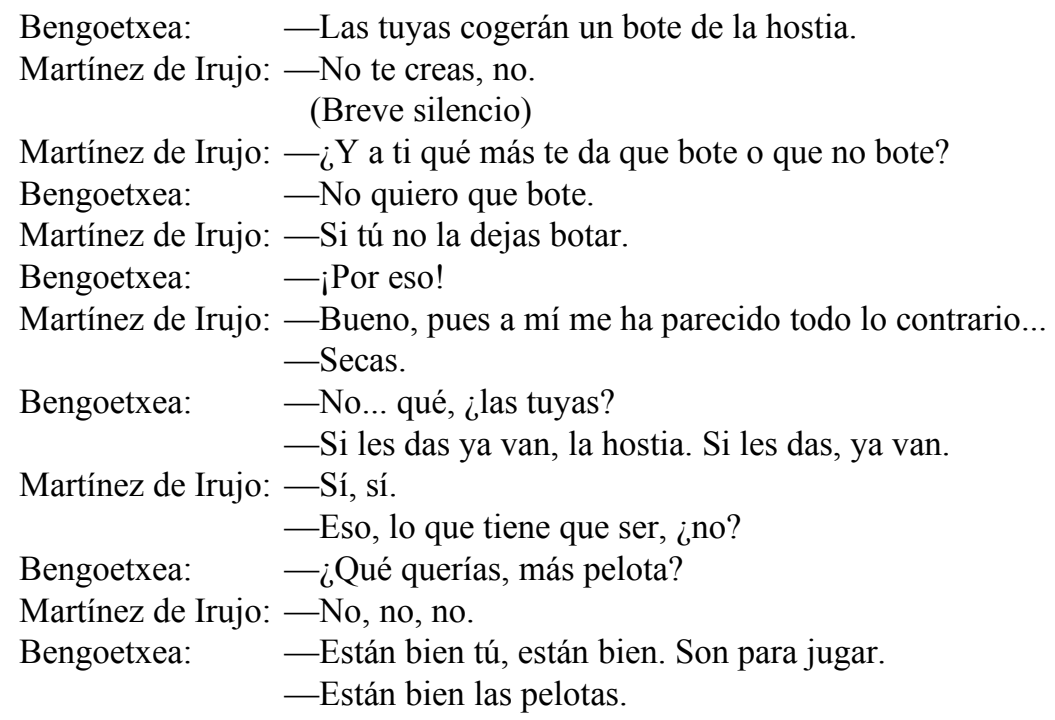

Esta conversación, que puede visualizarse en la película Pelota II y que se produjo después de la selección de material de una de las semifinales del manomanista del año 2013, ejemplifica lo que sucede una vez que las pelotas han sido elegidas. Las risas y conversaciones informales previas a la selección se tornan tras ella en

\footnotetext{
Santiago Moreno Cruzada, en su análisis del engaño en la caza (2019: 170 y ss.), ofrece un matiz a la propuesta de Ervin Goffman sobre la situación interactiva que sería interesante aplicar en este contexto. Cruzada defiende que las prácticas de engaño expanden y facilitan el "orden de interacción", anticipando y haciendo posible el encuentro "cara a cara": "el engaño sería el marco de interacción en sí mismo, sin necesariamente haber tenido, cazadores y animales, una copresencia inmediata, ya que precisamente lo que se busca con el engaño es que esa copresencia se produzca” (ib, 173). Así comprendido, el engaño encontraría un paralelo deportivo en el tanteo, al que refiero más adelante.
} 
desconfianza. Al elegir las pelotas, un pelotari está diciendo al otro cuáles cree que son sus debilidades y este no hace más que verlas reflejadas en las pelotas, que de algún modo las materializan. Atendiendo a las pelotas elegidas por el contrario, cada uno será capaz de colocarse en la posición del otro y mirarse desde allí. Ambos pelotaris se sentirán atrapados en la mirada del otro.

Este juego de perspectivas recuerda a aquel que Eduardo Viveiros de Castro encuentra en las cosmologías amazónicas y que le lleva a postular el "perspectivismo", un concepto que refiere a aquellas concepciones según las cuales "el mundo está compuesto por una multiplicidad de puntos de vista: todos los existentes son centros de intencionalidad, que aprehenden a los otros existentes según sus respectivas características y capacidades" (2010: 33). Los amazónicos habitan un mundo repleto de sujetos que ven al resto de seres en relación a su propia posición dentro de una especie de cadena trófica. Ante un encuentro con otra clase de seres, o incluso con uno que crees identificar fácilmente, averiguar cómo te ve el otro - como depredador o presa - es una de las máximas preocupaciones para saber cuál será tu suerte; esto es, cuál pasará a ocupar la posición de sujeto y cuál la de objeto.

Viveiros de Castro afirma que "el perspectivismo amerindio está asociado a dos características recurrentes en la Amazonía: la valorización simbólica de la caza y la importancia del chamanismo" (2004: 42). La caza ha sido también el modelo desde el que se ha pensado la pelota. Jorge Oteiza ya apreció que los vascos "juegan como cazadores y a cazarse, a quién resulta cazador y hombre, y quién como presa o animal dominado. A quién escapa del Laberinto y quién se queda,

juegan a minotauros de los nuestros y a teseos. Y el estilo que juegan, y se rebela en el frontón, es de trampas, perifrástica de huecos" (en Pelay Orozco, 1983: 24). Pelotaris y aficionados hablan constantemente de cazar al otro o haber sido cazado por él. La caza en el frontón consiste en descubrir los huecos, las debilidades del rival, para poder hacerse con él, dominarlo. Para ello, como en cualquier arte venatoria, la caza del otro pasa necesariamente por comprenderlo, por conocer sus intenciones, pero al mismo tiempo por ocultar las propias. Más allá de la apreciación de José Ortega y Gasset, que opinaba que "si el cazado fuese también y en la misma ocasión cazador, no habría caza", sino que estaríamos ante un esquema de lucha (1999: 35-36), el partido se concibe como un proceso de caza, en lo que consiste precisamente el tanteo; no solo la acumulación de puntos que compone el partido, sino también el procedimiento por medio del cual los pelotaris se ponen a prueba, se reconocen, y en definitiva se tantean.

En pelota el tanteo comienza ya con la selección de material. Serán las pelotas, con sus diferencias, las que obliguen a los pelotaris a tantearse, a colocarse en la posición del otro y verse desde ahí. Es lo que Roy Wagner denomina "reciprocidad de perspectivas", situación en la que cada uno es a la vez sujeto y objeto del otro (2018). Este intercambio de puntos de vista o transmutación de objeto y sujeto (object/subject shift) permite ocupar la mirada del otro para buscar el modo de burlarla y escapar así de la trampa perspectivista que tienden las pelotas. Hacerse con la pelota, encontrarla, por tanto, no se trata solo de ajustarse a las cualidades que supuestamente promueve, sino descubrir aquellas que esconde y que son su poder oculto. Quien sepa inventar lo que la pelota mantiene impensado, lo que no está previsto, tendrá más posibilidades de resolver a su favor el partido. Sacarle 
partido a la pelota es descubrir aquello que nadie esperaba que contuviera, llevarla más allá de su simple conformación, algo que, por efecto de recursividad o contrainvención, hace trascender también la del propio pelotari.

Esta cualidad contrainventiva de la reciprocidad de perspectivas es la que obvia el humanismo moderno cuando estandariza los objetos para reducir la impredecibilidad y racionalizar el deporte. Es la cualidad que intuye Serres al proponer el concepto de cuasiobjeto, o su reverso, el cuasisujeto, que implica que "el objeto se transforma en sujeto y viceversa" (1987: 182), y está implícita también en la cita que inicia este artículo y que dice que "será sujeto quien se encuentre activado o 'accionado' por el punto de vista" (Viveiros de Castro, 2004: 43). Nadie niega que esa dialéctica tenga lugar en un encuentro deportivo, en el que ambos jugadores son objetos y sujetos del otro al mismo tiempo. Competir, competere, es ir al encuentro una cosa de otra, y no parece difícil convenir que, en caso de tratarse de humanos, ese encuentro va a estar atravesado por las proyecciones que cada una de las partes haga de la otra y por las invenciones resultantes de las consideraciones mutuas. Más difícil es aceptar que ese encuentro pueda producirse o ser activado desde las cosas mismas. Hay, sin embargo, ontologías que así lo plantean y que muestran formas de subjetivación que difieren de las que produce la idealización moderna.

\section{El mundo de la pelota}

La etnografía ha ofrecido variados testimonios que, calificados como animistas, dan cuentan de la amplia experiencia humana generada gracias a la activa interpelación de las cosas. Piedras que abren la boca (Hallowell, 1960) o que saltan al regazo del paseante (Bird-David, 1999), montañas que expresan sus quejas (De la Cadena, 2010) o selvas que piensan (Kohn, 2013); otros mundos están poblados de seres que se dan a conocer, interactúan con humanos y se aprovechan de ellos. A pesar de su distancia, ahondar en estas ontologías y en las invenciones antropológicas que ellas han generado puede iluminar nuestro caso y ayudarnos a imaginar qué revela el hecho de que en pelota los objetos de juego no hayan sido estandarizados; qué ofrece este deporte a aquellos que en él se socializan. Sigamos para ello el intento de Nurit Bird-David por traducir a una mente positivista como la nuestra la importancia de los devaru o superpersonas para los Nayaka del sur de la India (1999).

Los devaru son entes del medio natural — piedras, plantas, animales u otrosque "asaltan" a las y los Nayaka durante sus actividades cotidianas y con los que estos inician una relación especial, de mutua dependencia. En determinadas ocasiones, los devaru poseen a los Nayaka, y se expresan públicamente, pasando a formar parte de la comunidad, que les reconoce como integrantes de la misma, en ocasiones incluso como parientes. Según Bird-David, los devaru ayudarían a los Nayaka a ahondar en su proceso de dividuación, es decir, en el reconocimiento de los vínculos sociales que los constituyen. Bird-David transforma en acción - dividuar - el concepto popularizado por Marilyn Strathern — dividuo - para contraponer la concepción melanesia de persona, que objetiva las relaciones y las hace visibles, a la concepción occidental, que las omite para pensarse como un ser 
único e indivisible, individuo. Pretende así mostrar Bird-David que los devaru son objetivaciones de las relaciones que las y los Nayaka establecen con el mundo y que les permiten dividuarse, es decir, no solo reconocerse como seres relacionales, sino atender y hacer conscientes los cambios que se producen en y entre los agentes con los que se relacionan y, por tanto, en sí mismos.

Un proceso análogo es el que vive el pelotari en su permanente reconocimiento de las pelotas. El que la pelota se conceptualice como un ser único, singular, obliga al pelotari a atender a sus diferencias y entrar con ellas en una relación de reconocimiento que va a replicarse en sus relaciones con otros agentes. Cuando el pelotari Martínez de Irujo tuvo que abandonar la pelota por una dolencia cardiaca, su máximo rival - Olaizola II - reconocía que su juego dependía en gran parte del de él, que habían coevolucionado juntos. Esa coevolución hizo de Martínez de Irujo el pelotari más fuerte del cuadro a pesar de que cuando debutó su mayor arma era la imaginación. Martín Ezkurra, el que fuera botillero de Retegi II y uno de los sabios de la pelota, había dicho de él que era "imaginativo hasta la temeridad". No obstante, su incapacidad para ganar a Olaizola, cuya astucia en demasiadas ocasiones se le anticipaba y le desbordaba, hizo que confiara cada vez más en su potencia y fue perdiendo gran parte de su inventiva. Esta transformación no hubiera sido posible, sin embargo, sin la transformación de las pelotas, que con Irujo aumentaron significativamente en bote y velocidad, lo que obligó a Olaizola II a transformar del mismo modo su manera de juego. Golpes que antes eran defensivos - como el de aire - han pasado a ser ofensivos, imprimiendo un ritmo de juego que nada tiene que ver con el de hace un par de décadas.

La pelota en definitiva permite al pelotari proyectarse de manera renovada en cada encuentro, además de reconocerse, transformarse y posicionarse en relación al resto de pelotaris. Este posicionamiento afectará asimismo a las pelotas mismas, ya que ciertas clases de pelota se priorizarán sobre otras y se irán modelando en paralelo a los cuerpos y habilidades de los pelotaris de cada época. Pero otros agentes entran también en la ecuación. Hace un par de años, la televisión dejó a Bengoetxea VI sin un tipo de pelotas que le gustaban mucho, las pelotas de cuero oscuro. La mala visibilidad que las mismas ofrecían en televisión ha provocado que ya no se pongan en el cestaño, lo que ha provocado las quejas continuadas de este pelotari en la selección de material, quien tendrá que inventar un nuevo modo de juego. Haciéndonos eco de las palabras de Donna Haraway: "A través de su contacto unos con otros, a través de sus 'aprehensiones' o sujeciones, los seres se constituyen unos a otros y a sí mismos. Los seres no preexisten a sus relaciones. Las 'aprehensiones' tienen consecuencias. El mundo es un nudo en movimiento" (2016: 17). Hay, sin embargo, formas de entender ese nudo, representaciones del mismo que otorgan agencia a unos seres y no a otros, y que tendrán consecuencias en el movimiento del mismo, en su conformación y deriva.

Mientras que en las sociedades autoproclamadas modernas los objetos pasan a formar parte de un paisaje inactivo (pasivo, silencioso) en el que los seres humanos dirimen sus diferencias desde una posición imaginadamente autoconstituida, en muchas sociedades otras, aunque se trate como en este caso de Internal Others (Candea, 2012) o indígenas occidentales (Cruzada, 2017), los objetos son explícitamente reconocidos como parte activa en la conformación de las personas y de su suerte, así como de los mundos que estas habitan. En el juego de pelota, las 
pelotas son definidas como "seres vivos", con su carácter y su temperamento, pudiendo ser caprichosas, elegantes, nobles, exigentes o violentas, entre otras muchas cosas. También pueden variar su humor en los diferentes momentos de juego: pueden estar alegres o apáticas, haberse quedado sin chispa, calentarse, o rebelarse contra el pelotari. Son seres vivos y como tales, impredecibles. Poseen personalidad y además tienen sus momentos, algo que afecta a las relaciones entre los diversos agentes que componen el "mundo de la pelota"; acertada denominación que adquiere aquí toda la fuerza de su literalidad. Muchos partidos han dado la vuelta gracias a una pelota. Miles de euros han cambiado de bolsillo por un cambio en el cestaño. El país vasco de la pelota nunca ha sido moderno.

\section{Conclusión}

Dentro de la simetría generalizada de Latour y otros, la distinción objeto-sujeto es irrelevante para entender la agencia, ya que esta está distribuida entre todos los actantes que participan en ella: "La acción no es una propiedad atribuible a los humanos, sino a una asociación de actantes" (2001: 218). Esto, como apunta Iñigo Galzacorta, no implica que los objetos se limiten "a servir a una meta previamente establecida a la que simplemente añaden eficacia. Por el contrario, (...) lo que ocurre más bien es que tanto en el propio uso de estos objetos, como previamente en el proceso de diseño, producción o conocimiento de los mismos, las metas, el significado de nuestra acción, la humanidad misma de quien se entrelaza con los objetos, resulta alterada, desplazada, traducida en y por el propio proceso" (2016: 362). Este artículo ha procurado mostrar cómo se produce eso en el caso de la pelota. Ocurre, sin embargo, que es imposible trazar a todos los actantes que participan en una acción y, como critica Tim Ingold a la propuesta latouriana, el cierre categorial que se impone sobre las cosas olvida su precariedad ontológica, ocultando la ecología de materiales y fuerzas de las que estas emergen (2012). Todo el mundo omite algo o a alguien, y de esas omisiones u obviaciones deriva, según Wagner, toda invención (1981).

La modernidad inventó los objetos omitiendo todo lo que en ellos había de particular, singularizante, y que dependía a partir de entonces del favor de los sujetos con los que aquellos entraran en contacto y que fueron inventados en paralelo a los primeros. La estandarización es la culminación material de la omisión de la singularidad de los objetos, que ha hecho prevalecer la omnipotencia del sujeto frente a sus encuentros o agenciamientos con las cosas, a las que ha creído poder objetivar de manera definitiva. Los objetos se convirtieron así en mercancía que podía ser sustituida sin comprometer al sujeto, que quedaba libre de las cargas morales del intercambio. El objeto era suyo, pero no le comprometía. Podía deshacerse de él en cualquier momento y reponerlo por otro exactamente igual, que le diera la misma satisfacción y prolongará su ficción de ser independiente del mismo, de no necesitarlo. Toneladas de basura se acumulan ahora gracias a esta ficción.

Pero esta ficción de la autonomía del sujeto, que inventa el objeto desechable, también tiene consecuencias en los procesos de subjetivación, ya que como teoriza Nikolas Rose, la subjetivación no se produce por medio de narrativas sino con 
objetos. A lo largo del artículo he intentado mostrar qué ofrece la singularidad de las pelotas al juego que le dan nombre. El que los objetos supuestamente imparciales no estén estandarizados genera sin duda incertidumbre. Los pelotaris no saben si lo que van a encontrarse va a responder a sus demandas y eso les inquieta. La imparcialidad de las pelotas puede ser cuestionada, pero en ningún caso genera indiferencia. Todos los pelotaris coinciden en que las pelotas no debieran estandarizarse. Más bien, lo consideran algo imposible dada su composición, que califican de "viva". Los peloteros saben que la estandarización es posible y algunos la promocionan, pero desde el mundo de la pelota esta se concibe como un sacrilegio. Que todas las pelotas suenen igual se les hace a los expertos un paisaje desértico, sin matices. Los que juegan con las pelotas saben además lo que estas les ofrecen: la posibilidad chamánica de mirarse desde el otro. Las pelotas dan acceso a las estrategias del contrario y a lo que él cree saber de las tuyas. También permiten descubrir nuevas formas de jugarlas y nuevas tácticas, inventarlas e inventarse en cada partido. Las pelotas son fuente de creatividad y de conocimiento de uno mismo. Pero, por encima de todo, las pelotas son un posible aliado, algo a lo que los pelotaris no parecen dispuestos a renunciar.

\section{Bibliografía}

Abrisketa, O. G. (2005): Pelota vasca: un ritual, una estética, Editores Muelle de Uribitarte.

Abrisketa, O. G. (2013): "Cuerpos desplazados. Género, deporte y protagonismo cultural en la plaza vasca", AIBR: Revista de Antropología Iberoamericana, 8(1), pp. 83-110.

Abrisketa, O. G. y S. C. Ripalda (2016): "La Apertura Ontológica de La Antropología Contemporánea", Revista de Dialectología y Tradiciones Populares 71 (1), pp. 101-128.

Abrisketa, O. G. y C. G. Grados (2018): “iIncorporar, encarnar, encorporar y/o corporear? Un ejercicio de traducción para pensar la agentividad de los cuerpos”, Mariluz Esteban y Jone Miren Hernández (coords.), Una mirada al siglo XXI desde la antropología vasca, pp. 231-247.

Bird-David, N. (1999): “'Animism’ Revisited: Personhood, Environment and Relational Epistemology”, Current Anthropology 40 (S1), pp. S67-S91.

Candea, M. y L. Alcayna-Stevens (2012): "Internal Others: Ethnographies of Naturalism", The Cambridge Journal of Anthropology 30 (2), pp. 36-47.

Cruzada, S. M. (2017): "Nosotros también somos indígenas: la vulnerabilidad del naturalismo en contextos occidentales de convivencia entre especies", Etnográfica. Revista do Centro em Rede de Investigação em Antropologia, 21(1), pp. 49-71.

Cruzada, S. M. (2019): Encuentros de vida y muerte. Antropología transespecie y mundos ampliados entre cazadores y animales en el suroeste extremeño (Tesis Doctoral, Universidad Pablo de Olavide, Sevilla).

De la Cadena, M. (2010): "Indigenous Cosmopolitics in the Andes: Conceptual Reflections beyond 'Politics", Cultural Anthropology 25 (2), pp. 334-370.

Domínguez Rubio, F. (2016): "On the discrepancy between objects and things: An ecological approach", Journal of Material Culture, 21(1), pp. 59-86. 
Galzacorta, I. (2016): "Siguiendo a los cuasi-objetos: Modernidad, inmanencia y trascendencia en Bruno Latour", Eikasia: revista de filosofia, (71), pp. 345-368.

Guttmann, A. (2004): From Ritual to Record: The Nature of Modern Sports, Columbia University Press.

Hallowell, A. I. (1960): Ojibwa Ontology, Behavior, and World View, Columbia University Press.

Haraway, D. J. (2016): Manifiesto de las especies de compañia, Sans Soleil.

Henare, A., M. Holbraad y S. Wastell (2007): Thinking through Things: Theorising Artefacts Ethnographically, Routledge.

Ingold, T. (2000): The perception of the environment: essays on livelihood, dwelling and skill. Psychology Press.

Ingold, T. (2012): "Toward an ecology of materials", Annual review of anthropology, 41, pp. 427-442.

Ishii, M. (2012): “Acting with Things: Self-Poiesis, Actuality, and Contingency in the Formation of Divine Worlds", HAU: Journal of Ethnographic Theory 2 (2), pp. 371-388.

Kohn, E. (2013): How Forests Think: Toward an Anthropology beyond the Human, Univ of California Press.

Kohn, E. (2015): “Anthropology of Ontologies”, Annual Review of Anthropology 44, pp. 311-327.

Latour, B. (1996): “On Actor-Network Theory: A Few Clarifications”, Soziale Welt, pp. 369-381.

Latour, B. (2001): La Esperanza de Pandora, Barcelona, Gedisa.

Latour, B. (2008): Reensamblar Lo Social: Una Introducción a La Teoría Del Actor-Red. $302.3 \mathrm{~L} 3$.

Law, J., y J. Urry (2004): “Enacting the social”, Economy and society, 33(3), pp. 390-410.

Lévi-Strauss, C. (2002): El Pensamiento Salvaje, Madrid, Fondo de Cultura Económica.

Messner, M. A. (1988): "Sports and male domination: The female athlete as contested ideological terrain", Sociology of sport journal 5.3, pp. 197-211.

Ortega y Gasset, J. (1999): “Sobre La Caza, Los Toros y El Toreo", Revista de Occidente, Madrid, Alianza Editorial.

Pedersen, M. A. (2007): "Talismans of Thought: Shamanist Ontologies and Extended Cognition in Northern Mongolia", in Thinking Through Things, pp. 151-176, Routledge.

Pelay Orozco, M. (1983): Pelota, Pelotari, Frontón, Poniente.

Serres, M. (1980): Le Parasite, Paris, Grasset.

Serres, M. (1987): Statues: le second livre des fondations, François Bourin.

Tirado, F. y M. Maureira (2016): "De Objetos y Extituciones: Nuevos Operadores de Lo Social”, OXÍMORA Revista Internacional de Ética y Politica, no. 8, pp. 112-130.

Varela, F. J., E. Thompson y E. Rosch (1992): El Cuerpo Presente: Las Ciencias Cognitivas y La Experiencia Humana, Gedisa.

Viveiros de Castro, E. (2004): "Perspectivismo y Multinaturalismo En La America Indígena", Surrales, Alexandre, Garcia Del Hierro, Pedro. Tierra Adentro. Territorio Indigena y Percepción Del Entorno. IWGIA. Lima Perú.

Viveiros de Castro, E. (2010): Metafisicas Caníbales: Líneas de Antropología Postestructural, Katz Editores.

Wagner, R. (1981): The Invention of Culture, University of Chicago Press.

Wagner, R. (2018): The Logic of Invention, Hau Books. 